\title{
Spherically Invariant Random Processes for Modeling Non-Gaussian Radar Clutter
}

\author{
Muralidhar Rangaswamy \\ Rome Laboratory/ERCE, Hanscom AFB, MA
}

September 16, 1993

\begin{abstract}
This investigation is motivated by the problem of modeling correlated non-Gaussian radar clutter. Experimental research has confirmed that radar clutter can have an extended tail under certain conditions. Since the Gaussian model fails to predict the extended tail behavior, non-Gaussian probability density functions (PDF) have been used for the first order PDF of the clutter. Usually, radars process $N$ samples at a time. Therefore, a complete statistical characterization would involve the ability to specify the joint PDF of $N$ correlated non-Gaussian random variables. This paper presents mathematically elegant and tractable techniques for specifying the joint PDF of $N$ correlated non-Gaussian random variables. The approach used in this paper is based on the theory of spherically invariant random processes (SIRP). Several important properties of SIRPs are summarized in this paper.
\end{abstract}

\section{Introduction}

This investigation is motivated by the problem of modeling correlated non-Gaussian radar clutter. For many years, radar systems had rather low resolution capabilities. The clutter echoes arising in such systems were approximated as the resultant of the returns from a large number of scatterers. Consequently, the central limit theorem was used to conclude that the statistics of such clutter was Gaussian. Such a model enabled the analysis and design of the optimal radar signal processor. In order to improve performance, high resolution radar systems were proposed. A high resolution radar system intercepts less clutter power owing to the small cell sizes. Consequently, the statistics of clutter encountered in high resolution radar systems exhibited departure from the Gaussian behavior. In particular, experimental research indicated that the clutter tends to have an extended tail. The extended tail behavior gives rise to large false alarm probabilities. The Gaussian model fails to predict the extended tail behavior. Deviations from the Gaussian behavior can be explained by the fact that the central limit theorem is not applicable for radar clutter encountered in high resolution radar systems. Therefore, non-Gaussian probability density functions (PDFs) were proposed to account for the large tail behavior of the clutter.

Commonly reported marginal non-Gaussian PDFs for the clutter are Weibull [1], log-normal $[2,3]$ and $K$ - distributions $[4,5,6]$. Second order statistics for these models have been reported in terms of autocorrelation functions or power spectral densities $[7,8]$. However, such models are lacking in their ability to provide a complete statistical characterization of the clutter. A complete statistical characterization of the clutter involves the specification of the joint PDF of $n$ random variables obtained by sampling the clutter process. A complete statistical characterization of the clutter is necessary for designing the optimal radar signal processor. While dealing with correlated non-Gaussian random variables, there are no unique analytical expressions for their joint PDF. This is due to the fact that not all higher order dependencies can be satisfactorily accounted for by a given joint PDF. However, in practice, the only information available is the first order PDF and the correlation function.

Spherically invariant random processes (SIRPs) provide a powerful mechanism for specifying the joint PDF of $n$ correlated non-Gaussian random variables because they enable the construction of the $n^{\text {th }}$ order PDF based on the first order PDF and correlation function. Hence, they offer an elegant and mathematically tractable approach for modeling correlated nonGaussian radar clutter. In this paper we use SIRPs for modeling correlated non-Gaussian radar clutter. We present several important properties of SIRPs and summarize the main results of [9] in this paper. $\mathrm{Fi}$ nally, we point out that the SIRP model for radar clutter is an extension of the so called compound model in that it enables the specification of the $n^{t h}$ order PDF.

\section{Background}

We present definitions and mathematical preliminaries in this section. We begin by defining the terms spherically invariant random vector (SIRV) and spherically invariant random process (SIRP). A random vector $\mathbf{Y}=\left[Y_{1}, Y_{2}, \ldots Y_{N}\right]^{T}$ is said to be an SIRV if its PDF has the form

$$
f_{\mathbf{Y}}(\mathbf{y})=k|\mathbf{\Sigma}|^{-\frac{1}{2}} h_{N}(p)
$$

where $p=(\mathbf{y}-\mathbf{b})^{T} \mathbf{\Sigma}^{-1}(\mathbf{y}-\mathbf{b}), \mathbf{b}$ is an $N \times 1$ vector, $\mathbf{\Sigma}$ is an $N \times N$ non-negative definite matrix, |.| denotes determinant, $k$ is a constant so that the volume under the PDF curve is unity and $h_{N}($.$) is a one$ dimensional, non-negative, real valued, monotonically 
decreasing function for all $N$. If every random vector obtained by sampling a process $y(t)$ is an SIRV regardless of the number of samples or sampling instants, the process $y(t)$ is said to be an SIRP. Observe that the PDF of eq (1) is a function of a non-negative quadratic form $p$.

The work of [10] gave rise to a representation theorem for SIRVs which states that a random vector is an SIRV if and only if it is equivalent to the product of a Gaussian random vector and an independent non-negative random variable. As a consequence of the representation theorem, it follows that $\mathbf{Y}=\mathbf{Z S}$ where $\mathbf{Z}=\left[Z_{1}, Z_{2}, \ldots, Z_{N}\right]^{T}$ is a Gaussian random vector and $S$ is a non-negative random variable with PDF $f_{S}(s)$ defined to be the characteristic PDF of the SIRV. Also, it can be shown from the representation theorem that $k=(2 \pi)^{-\frac{N}{2}}$ and

$$
h_{N}(p)=\int_{0}^{\infty} s^{-N} \exp \left(-\frac{p}{2 s^{2}}\right) f_{S}(s) d s .
$$

Furthermore, the covariance matrix of the SIRV, $\mathbf{\Sigma}$, can be made equal to the covariance matrix of the Gaussian random vector by requiring the mean square value of $S, E\left[S^{2}\right]$, to be unity. Thus, the PDF of an SIRV is completely determined by the specification of a mean vector, $\mathbf{b}$, a covariance matrix, $\boldsymbol{\Sigma}$, and a characteristic first order PDF, $f_{S}(s)$. Thus, given $\mathbf{b}$ and $\Sigma$, all that is needed to determine the SIRV PDF is the specification of $h_{N}(p)$. When $f_{S}(s)=\delta(s-1)$, eq (2) results in $h_{N}(p)=\exp \left(-\frac{p}{2}\right)$. The resulting PDF using eq (1) is simply the multivariate Gaussian PDF.

\subsection{Specification of the SIRV PDF}

Many non-Gaussian marginal PDFs are admissible as SIRVs. Several techniques are available for determining $h_{N}(p)$ for these non-Gaussian PDFs. These techniques are briefly outlined below. The interested reader is referred to [9] for details. The most straightforward technique is to use the representation theorem. More precisely, given a particular first order PDF, we use eq (1) with $N=1$ and determine $h_{1}(p)$. Then, we use eq (2) with $N=1$ and solve for $f_{S}(s)$. Finally, eq (2) is used with the desired $N$ for determining the corresponding $h_{N}(p)$. The SIRV PDF is obtained from eq $(1)$. In many instances, $f_{S}(s)$ is not known in closed form. Also, the integral of eq (2) can be difficult to evaluate. In either event, alternate means for specifying $h_{N}(p)$ must be examined.

We point out that $h_{1}($.$) and h_{2}($.$) are related to the$ first order and second order SIRV PDFs, respectively. An approach based on differentiating $h_{1}($.$) and h_{2}($. lends itself for determining $h_{N}($.$) for all N$. For convenience, we consider eq (2) with $p$ replaced by $w$. It has been noted in [9] that differentiating $h_{N}(w)$ with respect to $w$ results in

$$
\frac{d}{d w}\left[h_{N}(w)\right]=-\frac{1}{2} h_{N+2}(w)
$$

More precisely, starting with $N=1$ and $N=2$, eq (3) can be rewritten to obtain the following pair of recur- rence relations.

$$
\begin{aligned}
& h_{2 N+1}(w)=(-2)^{N} \frac{d^{N}}{d y^{N}}\left[h_{1}(w)\right] \\
& h_{2 N+2}(w)=(-2)^{N} \frac{d^{N}}{d w^{N}}\left[h_{2}(w)\right] .
\end{aligned}
$$

$\mathrm{Eq}$ (4) provides a mechanism for relating higher order PDFs with those of lower order. In particular, starting from $h_{1}($.$) and h_{2}($.$) , the PDFs of all odd and$ even $N$ can be determined by using eq (4). Finally, in order for $h_{N}($.$) obtained from eq (4) to result in$ valid PDFs, $h_{1}($.$) and h_{2}($.$) must belong to a class of$ monotonically decreasing functions. In addition, their successive derivatives must alternate between negative and positive functions which are increasing and decreasing, respectively.

In some instances, the first order characteristic function $\phi(\omega)$ corresponding to a particular first or der PDF may be known. In such cases, it has been shown [10] that $h_{N}(p)$ is given by

$$
h_{N}(p)=(\sqrt{p})^{1-\frac{N}{2}} \int_{0}^{\infty} \omega^{\frac{N}{2}} \phi(\omega) J_{\frac{N-2}{2}}(\omega \sqrt{p}) d \omega
$$

where $J_{\alpha}($.$) is the Bessel function of order \alpha . \mathrm{Eq}(5)$ is simply the Hankel transform of $\phi(\omega)$ of order $\frac{N}{2}-$ 1. Thus, $h_{N}($.$) corresponding to a given \phi(\omega)$ can be obtained by using a table of Hankel transforms.

\subsection{Properties of SIRPs}

Several attractive properties of the Gaussian random process readily extend to SIRPs on account of the representation theorem. These properties are summarized in this section. Proofs of these properties are available in [9].

1. The PDF of an SIRV is uniquely determined by the specification of a mean vector, a covariance matrix and a characteristic first order PDF.

2. The PDF of an SIRV is internally consistent.

3. If a random vector $\mathbf{X}$ is an SIRV having characteristic PDF $f_{S}(s)$, then every linear transformation of the form $\mathbf{Y}=\mathbf{A X}+\mathbf{b}$ results in an SIRV with the same characteristic PDF. This property is called the closure property under linear transformation [10].

4. In minimum mean square error estimation problems, given a set of data, SIRVs are found to result in linear estimators $[10]$.

5. While the sum of two independent Gaussian random vectors is Gaussian, the same result does not hold for two independent SIRVs in general. This result holds only when the covariance matrix of one SIRV is to within a multiplicative constant of that of the other [10].

6. An SIRP is Markov if and only if its autocorrelation function has the form $R\left(t_{1}, t_{2}\right)=$ $\operatorname{aexp}\left(-\alpha\left|t_{1}-t_{2}\right|\right)[9]$. 
7. The Kalman filter for SIRPs is identical to the Kalman filter for the Gaussian case [9].

8. Components of an SIRV can be statistically independent if and only if the SIRV is Gaussian [9].

9. An ergodic SIRP is necessarily Gaussian [9].

10. The maximum likelihood estimate of the covariance matrix of an SIRV is the sample covariance matrix [9].

\section{SIRP Model for Radar Clutter}

We discuss the SIRP approach for modeling correlated non-Gaussian radar clutter in this section. Specifically, we deal with coherent radar clutter. Predetection radar clutter is a bandpass process and admits a representation of the form

$$
y(t)=\operatorname{Re}\left\{\tilde{y}(t) \exp \left(j \omega_{0} t\right)\right\}
$$

where $\tilde{y}(t)=y_{c}(t)+j y_{s}(t)$ is the complex envelope of the clutter process whose in-phase and quadrature phase components are, $y_{c}(t)$ and $y_{s}(t)$, respectively and $\omega_{0}$ is a known carrier frequency. The statistics of the bandpass process $y(t)$ can be determined in terms of those of the process $\tilde{y}(t)$. Therefore, we work with $N$ complex samples or $2 N$ quadrature components obtained by sampling $\tilde{y}(t)$.

A complete statistical description of the clutter process is obtained by specifying the joint PDF of the $N$ complex samples or the $2 N$ quadrature components. The joint PDF enables us to derive the optimal radar signal processor for a given scenario. Since the clutter is apt to change in space and time, it is necessary to have available a library of joint PDFs so that a suitable PDF from this library can be used for approximating a given clutter scenario. Performance of the optimal radar signal processor cannot be determined analytically in general. Therefore, computer simulation is necessary. This requires the ability for efficient computer generation procedures for the clutter scenarios described by the library of PDFs. Finally, since the background clutter is unknown a priori, a procedure which approximates the PDF underlying a set of experimental clutter data is necessary. These issues have been addressed in considerable detail in [9]. We summarize the main results from [9] here.

We assume that $\tilde{y}(t)$ is an SIRP which implies that $y_{c}(t)$ and $y_{s}(t)$ are jointly SIRP. Let $\mathbf{Y}=$ $\left[\mathbf{Y}_{c}^{T} \mathbf{Y}_{s}^{T}\right]^{T}$ where $\mathbf{Y}_{c}=\left[Y_{c 1}, Y_{c 2}, \ldots Y_{c N}\right]^{T}$ and $\mathbf{Y}_{s}=$ $\left[Y_{s 1}, Y_{s 2}, \ldots Y_{s N}\right]^{T}$ denote the vector of quadrature components obtained by sampling $\tilde{y}(t)$. It is further assumed that the process $y(t)$ is wide sense stationary. The wide sense stationarity assumption enables the specification of the mean vector and covariance matrix of $\mathbf{Y}$. The PDF of $\mathbf{Y}$ is determined by the specification of $h_{2 N}(p)$. The results of section 2 are applicable for this problem with with $N$ replaced by $2 N$. The techniques presented in section 2.1 are used to construct a variety of joint PDFs. We present one example based on each approach outlined in section 2.1. and merely list other PDFs obtained.
As mentioned before, it is sometimes necessary to use eq (4) for obtaining $h_{2 N}($.$) . To facilitate the use$ of eq (4), we point out that $h_{2}($.$) is related to the first$ order envelope PDF. More precisely,

$$
h_{2}\left(\frac{r^{2}}{\sigma^{2}}\right)=\frac{\sigma^{2}}{r} f_{R}(r)
$$

where $f_{R}(r)$ is the PDF of $R=\sqrt{Y_{c i}^{2}+Y_{s i}^{2}}, i=$ $1,2, \ldots, N$ and $\sigma^{2}=\frac{1}{2} E\left[R^{2}\right]$.

3.1 Approach based on the Characteristic PDF

The K-distributed envelope PDF is popularly used for modeling sea clutter returns [6] and is given by

$$
f_{R}(r)=\frac{2 b}{\Gamma(\alpha)}\left(\frac{b r}{2}\right)^{\alpha} K_{\alpha-1}(b r) u(r)
$$

where $\alpha$ is the shape parameter of the distribution, $b$ denotes the scale parameter of the distribution, $K_{N}(t)$ is the $N^{\text {th }}$ order modified Bessel function of the second kind and $u(r)$ is the unit step function. The PDF of the quadrature components arising from the $\mathrm{K}$-distributed envelope PDF and an independent uniform phase is given by [9]

$$
\begin{gathered}
f_{Y_{\mathrm{c}}}(y)=f_{Y,}(y) \\
=\frac{2 b}{\Gamma(\alpha) \sqrt{2 \pi 2^{\alpha}}}|b y|^{\alpha-\frac{1}{2}} K_{\frac{1}{2}-\alpha}(b|y|) \quad-\infty<y<\infty .
\end{gathered}
$$

The characteristic PDF, $f_{S}(s)$, for the K-distribution is [9]

$$
f_{S}(s)=\frac{2 b}{\Gamma(\alpha) 2^{\alpha}}(b s)^{2 \alpha-1} \exp \left(-\frac{b^{2} s^{2}}{2}\right) u(s) .
$$

Using eq (2) with $N$ replaced by $2 N$ it follows that [9]

$$
h_{2 N}(p)=\frac{b^{2 N}}{\Gamma(\alpha)} \frac{(b \sqrt{p})^{\alpha-N}}{2^{\alpha-1}} K_{N-\alpha}(b \sqrt{p}) .
$$

Other joint PDFs obtained in [9] using this approach correspond to the Student-T, Laplace, Cauchy, and mixture of Gaussian distributions.

\subsection{Approach Based on the First order Envelope PDF}

The Weibull distributed envelope PDF is given by

$$
f_{R}(r)=a b r^{b-1} \exp \left(-a r^{b}\right) \quad(0 \leq r \leq \infty) .
$$

where $a$ is the scale parameter and $b$ is the shape parameter. The characteristic PDF for the Weibull envelope PDF is not known in closed form except for some particular values of the shape parameter. Therefore, we use eq (4) to obtain $h_{2 N}(p)$ for this example. Using eq (6), it follows that

$$
\begin{gathered}
h_{2}(w)=a b \sigma^{b} w^{\frac{b}{2}-1} \exp \left(-a \sigma^{b} w^{\frac{b}{2}}\right) \\
=(-2) \frac{d}{d w}\left[\exp \left(-A w^{\frac{b}{2}}\right)\right]
\end{gathered}
$$


where $A=a \sigma^{b}$. Using eq (4), it can be shown that [9]

$$
h_{2 N}(w)=\sum_{k=1}^{N} C_{k} w^{\frac{k b}{2}-N} \exp \left(-A w^{\frac{b}{2}}\right)
$$

where

$$
C_{k}=\sum_{m=1}^{k}(-1)^{m+N} 2^{N} \frac{A^{k}}{k !}\left(\begin{array}{c}
k \\
m
\end{array}\right) \frac{\Gamma\left(1+\frac{m b}{2}\right)}{\Gamma\left(1+\frac{m b}{2}-N\right)} .
$$

The Weibull envelope PDF is admissible for characterization as an SIRV for values of $b$ less than or equal to 2 . This is due to the fact that $h_{2}(w)$ and its derivatives fail to satisfy the monotonicity condition for other values of $b$. However, this is not a serious restriction for the point of view of radar clutter modeling because the Weibull envelope PDF is of interest in modeling large tailed clutter. Such a situation arises only when $0<b \leq 2$. Finally, $h_{2 N}(p)$ is obtained from eq (14) by replacing $w$ by $p$. Multivariate PDFs corresponding to the Generalized Rayleigh, Chi, Generalized Gamma and Rician distributions were obtained [9] using this approach.

\subsection{Approach Using the Characteristic Function}

We consider the Rician envelope PDF, arising from a non-zero mean complex Gaussian process, given by

$$
f_{R}(r)=\frac{r}{\alpha^{2}} \exp \left[-\frac{\left(r^{2}+a^{2}\right)}{2 \alpha^{2}}\right] I_{0}\left(\frac{a r}{\alpha^{2}}\right) .
$$

The characteristic function corresponding to the Rician envelope PDF is [9]

$$
\phi(\omega)=\exp \left(-\frac{\omega^{2} \alpha^{2}}{2}\right) J_{0}\left(\frac{\omega a}{\alpha^{2}}\right) .
$$

Using eq (5) with $N$ replaced by $2 N$, it is seen that [9]

$$
\begin{gathered}
h_{2 N}(p)= \\
\frac{\alpha^{2 N+2}}{2^{2 N+1} \Gamma(N)} \sum_{m=0}^{\infty} \frac{\Gamma(m+N+1)}{m ! \Gamma(m+1)}\left(\frac{-a^{2}}{2 \alpha^{6}}\right)^{m} \\
\times F\left(-m,-m ; N ; \frac{p \alpha^{4}}{a^{2}}\right)
\end{gathered}
$$

where $F(., . ; . ;$.$) is the four parameter hypergeo-$ metric function. Other multivariate PDFs obtained in [9] using this approach correspond to the Gaussian, Laplace, K-distributed, Cauchy and Student-T PDFs.

4 SIRPs for Radar Clutter Simulation

The performance of a radar signal processor operating in a non-Gaussian clutter background cannot be determined analytically in general. Therefore, there is a need for computer generation of the clutter. The problem of computer generation of correlated nonGaussian radar clutter is equivalent to the problem of generating random variables with a jointly specified marginal PDF and covariance matrix. While the problem of generating random sequences with either a specified PDF or prescribed covariance function has been well treated [11], the joint problem has received limited attention.

In general, it has been possible to control either the PDF or the correlation function but not both simultaneously. Previous attempts $[2,12,7,13,14]$ to address the problem of generating random sequences with jointly specified marginal PDF and covariance function have not been successful because the procedures proposed therein made use of zero memory nonlinear (ZMNL) transformations on a correlated Gaussian sequence to obtain the desired non-Gaussian sequence. Consequently, the covariance matrix of the non-Gaussian sequence was related to that of the Gaussian sequence in a rather complicated manner. Hence, given a certain covariance matrix for the nonGaussian sequence, it was not possible to determine the corresponding covariance matrix of the Gaussian sequence. Furthermore, not all nonlinear transformations gave rise to non-negative definite covariance matrices at their outputs $[15,11]$. Finally, the output of the ZMNL transformation is bandlimited if and only if the input Gaussian process is bandlimited and the nonlinearity is a polynomial. Thus, using ZMNL transformations on a correlated Gaussian sequence does not offer a practical solution to the joint problem. The techniques presented in [9] using SIRPs successfully overcome the drawbacks of the previous efforts. This is due to the fact that SIRPs belong to the class of exogenous product models for radar clutter, which allows for independent control of the marginal PDF and correlation function.

Two canonical simulation schemes have been proposed in [9] for generating correlated non-Gaussian radar clutter which can be described by SIRPs. The first scheme is applicable for those SIRV PDFs for which the characteristic PDF, $f_{S}(s)$, is known in closed form. The second scheme is used for those SIRV PDFs for which $f_{S}(s)$ is unknown. Details of the two procedures are available in [9].

\section{Distribution Approximation of Radar Clutter Using SIRPs}

In practice, the clutter PDF encountered in radar signal processing is not known a priori. Consequently, a scheme that approximates the clutter PDF based on a set of measured data is necessary. Currently, available tests such as the Kolmogorov-Smirnov test and the Chi-Square test address the problem of goodnessof-fit for random data. In particular, these tests provide information about whether a set of random data is statistically consistent with a specified distribution, to within a certain confidence level. However, if the specified distribution is rejected, these tests cannot be used for approximating the underlying PDF of the random data. Moreover, these tests require large sample sizes for reliable results.

A new graphical procedure for approximating the univariate PDF underlying a set of random data was developed in [16]. This algorithm works well for small sample sizes (typically 100 samples). The algorithm developed in [16] was extended in [9] to the problem of distribution approximation of radar clutter which 
can be modeled as an SIRP. It has been shown in [9] that the PDF of an SIRV is uniquely characterized by the knowledge of the PDF of $p$. In particular, it was shown in [9] that the PDF of $p$ is given by

$$
f_{P}(p)=\frac{p^{\frac{N}{2}}-1}{2^{\frac{N}{2}} \Gamma\left(\frac{N}{2}\right)} h_{N}(p) u(p)
$$

Furthermore, the PDF of $p$ is invariant to $\mathbf{b}$ and $\boldsymbol{\Sigma}$. Also, the maximum likelihood estimates of $\mathbf{b}$ and $\boldsymbol{\Sigma}$ are, the sample mean and the sample covariance matrix, respectively [9]. Consequently, the multivariate distribution approximation problem for SIRVs was reduced to an equivalent univariate distribution approximation problem involving the PDF of $p$. This enabled the extension of the algorithm of [16] to SIRPs. Details of the distribution approximation procedure can be found in [9].

\section{Conclusions}

This work presents an overview of the results obtained in [9] for the problem of correlated nonGaussian radar clutter modeling, simulation and distribution approximation. As a consequence of this work, it was possible to develop the optimal radar signal processor operating in background clutter characterized by SIRPs $[17,18]$. A significant performance improvement over the conventional radar receiver was observed in $[17,18]$.

\section{Acknowledgments}

This work was supported by AFOSR and was carried out while Dr. Rangaswamy was a research associate of the National Research Council at Rome Laboratory, Hanscom AFB. The author gratefully acknowledges the many interesting discussions with Drs. J.H. Michels, D.D. Weiner and Mr. J.F. Lennon.

\section{References}

(1] M. Sekine, T. Musha, Y. Tomita, T. Hagisawa, T. Irabu, and E. Kiuchi, "On Weibull distributed weather clutter," IEEE Trans. on Aerospace and Electronic Systems, vol. AES-15, pp. 824-828, 1979.

[2] A. Farina, A. Russo, and F. Studer, "Coherent radar detection in log-normal clutter," IEE Proc.F, Commun., Radar, $\mathcal{G}$ Signal Process., vol. 133, (1), pp. 39-54, 1986.

[3] E. Conte and M. Longo, "On a coherent model for log-normal clutter," IEE Proc.F, Commun., Radar, \& Signal Process., vol. 134, (2), pp. 198201,1987 .

[4] S. Watts and K. Ward, "Spatial correlation in Kdistributed sea clutter," IEE Proc.F, Commun., Radar, \& Signal Process., vol. 134, (6), pp. 526532,1987 .

[5] S. Watts, "Radar detection prediction in Kdistributed sea clutter and thermal noise," IEEE Trans. on Aerospace and Electronic Systems, vol. AES-23, pp. 40-45, 1987.
[6] E. Jakeman and P. Pusey, "A model for nonRayleigh sea echo," IEEE Trans. on Antennas and Propagation, vol. AP-24, pp. 806-814, 1976.

[7] G. Li and K.-B. Yu, "Modeling and simulation of coherent Weibull clutter," IEE Proc.F, Commun., Radar, \& Signal Process., vol. 136, (1), pp. 2-12, 1989.

[8] C. Hawkes and S. Haykin, "Modeling of clutter for coherent pulsed radar," IEEE Trans. on Information Theory, vol. IT-21, pp. 703-707, 1975.

[9] M. Rangaswamy, Spherically Invariant Random Processes for Radar Clutter Modeling, Simulation and Distribution Identification. PhD thesis, Syracuse University, 1992.

[10] K. Yao, "A representation theorem and its applications to spherically invariant random processes," IEEE Trans. on Information Theory, vol. IT-19, pp. 600-608, 1973.

[11] B. Liu and D. Munson, "Generation of a random sequence having a jointly specified marginal distribution and autocovariance function," IEEE Trans. on Acoust., Speech, Signal Processing, vol. ASSP-6, pp. 973-983, 1982.

[12] A. Farina, A. Russo, F. Scannapieco, and S. Barbarossa, "Theory of radar detection in coherent Weibull clutter,"IEE Proc.F, Commun., Radar, $\mathcal{E}$ Signal Process., vol. 134, (2), pp. 174-190, 1987.

[13] W. Szajnowski, "The generation of correlated Weibull clutter for signal detection problems," IEEE Trans. on Aerospace and Electronic Systems, vol. AES-13, pp. 536-540, 1977.

[14] P. Peebles, "The generation of correlated lognormal clutter for radar simulation," IEEE Trans. on Aerospace and Electronic Systems, vol. AES7, pp. 1215-1217, 1971.

[15] G. Wise, A. Traganitis, and J. Thomas, "The effect of a memoryless nonlinearity on the spectrum of a random process," IEEE Trans. on Information Theory, vol. IT-23, pp. 84-89, 1977.

[16] A. Ozturk, "A new method for univariate and multivariate distribution identification." Submitted for publication to JASA.

[17] M. Rangaswamy, D. D. Weiner, and J. H. Michels, "Innovations based detection algorithm for correlated non-Gaussian random processes," in Proceedings of the Sixth IEEE SSAP Workshop, (Victoria, B.C.), 1992.

[18] M. Rangaswamy, D. D. Weiner, and J. H. Michels, "Multichannel detection for correlated non-Gaussian random processes based on innovations," in Proceedings of the SPIE international symposium on optical engineering and photonics in aerospace and remote sensing, (Orlando, FL), 1993. 